\title{
Concomitant intraductal papillary mucinous neoplasm and neuroendocrine tumor of the pancreas
}

\author{
MITSUAKI ISHIDA $^{1}$, HISANORI SHIOMI ${ }^{2}$, SHIGEYUKI NAKA ${ }^{2}$, TOHRU TANI $^{2}$ and HIDETOSHI OKABE ${ }^{1}$ \\ ${ }^{1}$ Department of Clinical Laboratory Medicine and Division of Diagnostic Pathology; \\ ${ }^{2}$ Department of Surgery, Shiga University of Medical Science, Otsu, Shiga, Japan
}

Received July 15, 2012; Accepted September 25, 2012

DOI: 10.3892/ol.2012.952

\begin{abstract}
Intraductal papillary mucinous neoplasm (IPMN) and neuroendocrine tumor (NET) of the pancreas are rare tumors and their association is not expected to be frequent. However, certain studies have suggested that the concomitant occurence of these tumors may be more frequent than previously thought. In the current study, we describe a case of concomitant occurrence of IPMN and NET of the pancreas and review the clinicopathological features of previously published cases and the current one. A 68-year-old female was incidentally found to have dilatation of the main pancreatic duct. A distal pancreatectomy was performed under the clinical diagnosis of IPMN. Histopathological analysis revealed concomitant IPMN (low-grade) and NET G1 of the pancreas. Review of the clinicopathological features of the 15 previously reported cases of concomitant IPMN and NET of the pancreas as well as the present one indicated that: i) this condition mainly affects middle-aged females; ii) the main symptom is abdominal or back pain, or no symptoms; iii) a hormone production symptom was observed in only one case; iv) the most common degree of dysplasia of IPMN is low grade; and v) the size of the NET is not particularly large (average $15.1 \mathrm{~mm}$ ), although the clinical behavior is not always indolent (metastasis was observed in 3 cases). It is well known that IPMNs are associated with a high incidence of extrapancreatic malignancies, including colorectal and gastric carcinomas. Concomitant pancreatic NET and extrapancreatic malignancies may occur, therefore, systemic surveillance of extrapancreatic neoplasms and detection of concomitant NETs of the pancreas are necessary for patients with IPMN.
\end{abstract}

Correspondence to: Dr Mitsuaki Ishida, Department of Clinical Laboratory Medicine and Division of Diagnostic Pathology, Shiga University of Medical Science, Tsukinowa-cho, Seta, Otsu, Shiga 520-2192, Japan

E-mail: mitsuaki@belle.shiga-med.ac.jp

Key words: intraductal papillary mucinous neoplasm, neuroendocrine tumor, pancreas

\section{Introduction}

Intraductal papillary mucinous neoplasm (IPMN) is a rare intraductal epithelial neoplasm composed of mucin-producing cells arising in the main pancreatic duct or its branches (1). IPMNs are estimated to account for $1-3 \%$ of exocrine pancreatic neoplasms and $20 \%$ of all cystic neoplasms of the pancreas, and the incidence of this disease is considered to be increasing (1). The subtypes of IPMNs are recognized as main-duct and branch-duct types by macroscopic examination, and noninvasive IPMNs are classified into three categories on the basis of cytoarchitectural atypia: low-, intermediate- and high-grade dysplasia (1).

Pancreatic neuroendocrine neoplasms are uncommon and represent $1-2 \%$ of all pancreatic neoplasms (2). According to the recent World Health Organization Classification of the Digestive System, neuroendocrine neoplasms are classified into three categories: neuroendocrine tumor (NET) G1 and G2 and neuroendocrine carcinoma (NEC; NET G3) (2).

IPMN and NET of the pancreas are rare tumors and their association is not expected to be frequent. However, certain studies have suggested that the concomitant occurrence of these tumors may be more frequent than previously thought (3). In the current study, we describe a case of concomitant occurrence of IPMN and NET of the pancreas, and review the clinicopathological features of previously reported cases and the current one. The study was approved by the Ethics Committee of Shiga University of Medical Science, Shiga, Japan. Informed consent was obtained from the patient.

\section{Patient and methods}

Case report. A 68-year-old Japanese female with a past history of autoimmune hepatitis was incidentally found to have dilatation of the main pancreatic duct, measuring $\sim 5 \mathrm{~mm}$, in the pancreas tail by computed tomography (CT; Fig. 1). No other tumorous lesions were detected in the pancreas and other visceral organs by CT. No clinical symptoms of hormone overproduction were present. Under the clinical diagnosis of main-duct type IPMN, a distal pancreatectomy was performed.

The postoperative course has been uneventful, and no tumor recurrence has been observed during three years of medical follow-up. 


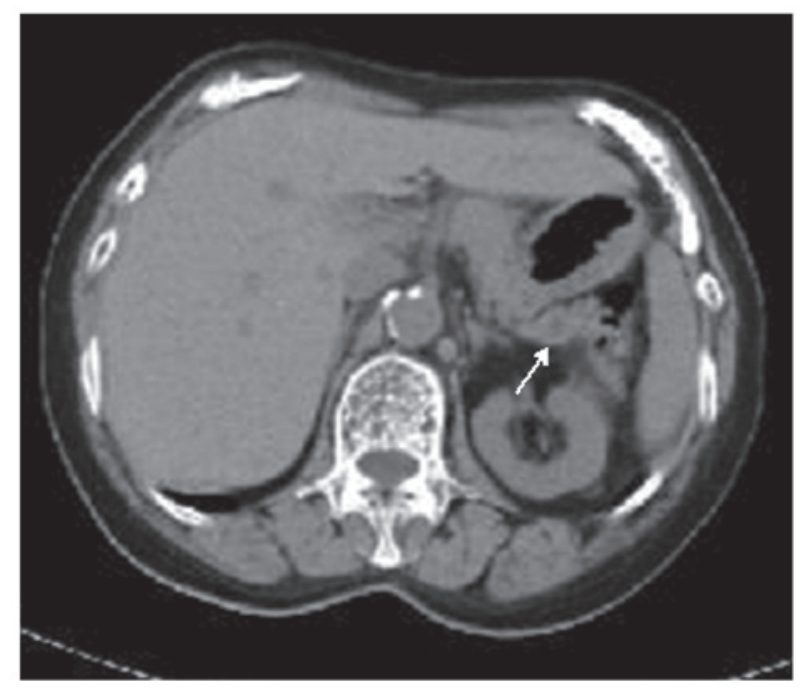

Figure 1. Dilatation of the main pancreatic duct in the pancreas tail (arrow).

Materials and methods. The formalin-fixed, paraffinembedded tissue blocks of the resected pancreas specimens were cut into $3-\mu \mathrm{m}$ thick sections, deparaffinized and rehydrated. Each section was stained with hematoxylin and eosin and then used for immunostaining. Immunohistochemical analyses were performed using an autostainer (Benchmark XT system; Ventana Medical System, Tucson, AZ, USA) according to the manufacturer's instructions. The following primary antibodies were used: a mouse monoclonal antibody against $\alpha$-internexin (2E3; Lab Vision, Freemont, CA, USA), a mouse monoclonal antibody against chromogranin A (DAK-A3; Dako Cytomation, Glostrup, Denmark), a rabbit polyclonal antibody against gastrin (Dako Cytomation), a rabbit polyclonal antibody against glucagon (Dako Cytomation), a mouse monoclonal antibody against insulin (Z006; Nichirei Bioscience, Tokyo, Japan), a mouse monoclonal antibody against Ki-67 (MM1; Novocastra Laboratories, Ltd., Newcastle-upon-Tyne, UK), a mouse monoclonal antibody against peripherin (PJM50; Novocastra Laboratories, Ltd.), a rabbit polyclonal antibody against somatostatin receptor type 2a (SSTR2a; Gramsch Laboratories, Schwabhausen, Germany) and a mouse monoclonal antibody against synaptophysin (27G12; Novocastra Laboratories, Ltd.).

\section{Results}

Histopathological study of the resected pancreas tissue revealed dilatation and intraductal papillary proliferation of the main pancreatic duct (Fig. 2A). The epithelial cells that showed intraductal papillary proliferation were columnar and had mucin in the cytoplasm and small round nuclei with inconspicuous nucleoli (Fig. 2B). Mitotic figures were rarely observed. No invasive growth was noted. These histopathological features corresponded to IPMN with low-grade dysplasia. Well-circumscribed neoplastic growth was present in the pancreas adjacent to the IPMN (Fig. 2A and C). Trabecular growth of the neoplastic cells with eosinophilic cytoplasm and bland nuclei with inconspicuous nucleoli was observed accompanied by fibrosis (Fig. 2C and D). Mitotic figures were rarely identified ( $<1 / 10$ high-power fields). No vascular invasion was noted.

Immunohistochemical analyses revealed that the neoplastic cells showing trabecular growth were diffusely positive for synaptophysin and chromogranin A (Fig. 3A). Glucagon was expressed in approximately half of the tumor cells (Fig. 3B), and a few insulin-positive tumor cells were also observed. However, gastrin positivity was not observed in any of the tumor cells. The Ki-67 labeling index was $<1 \%$. SSTR2a immunostaining was diffusely positive in the cell membrane of the tumor cells (Fig. 3C) and was score 3 according to the scoring system reported by Volante et al (4). No peripherin or $\alpha$-internexin expression was observed in the tumor cells.

According to these histopathological and immunohistochemical findings, an ultimate diagnosis of concomitant IPMN (low-grade dysplasia) and NET G1 of the pancreas was made.

\section{Discussion}

In the current study, we describe a case of concomitant IPMN and NET of the pancreas. Table I summarizes the clinicopathological features of the 15 previously reported cases of concomitant IPMN and NET $(3,5-9)$ as well as the present case. This condition mainly affects middle-aged females (average age, 63.1 years; range, 40-76 years; male/female ratio, 5:11). The main symptoms are abdominal or back pain (8 cases), no symptoms (5 cases) or weight loss (5 cases). A hormone production symptom (hypoglycemia) was observed in only one case. The most common degree of dysplasia of IPMN was low grade, however, high-grade dysplasia was also present ( 2 cases). The size of the NETs were not particularly large (average, $15.1 \mathrm{~mm}$; range, 3-30), however, the clinical behavior was not always indolent. Metastasis of the NET was observed in 3 cases and one of these cases succumbed to NET; the histopathology of this case was NEC (NET G3). The preoperative clinical diagnosis was variable; IPMN in 7 cases and concomitant in 6 cases. Therefore, detailed pathological analysis of the resected pancreas tissue is required to indicate adequate treatment since metastasis of the NET may occur in some patients with concomitant IPMN and NET, even in those with small-sized lesions.

Somatostatin is an acidic polypeptide that inhibits cell proliferation and differentiation (10). The physiological action of somatostatin is initiated by its interaction with a family of receptors consisting of five different subtypes, SSTR1-5 (11). Somatostatin analogs (including octreotide) bind to the SSTRs, particularly SSTR2a, which is the most widely expressed subtype in NETs (4). A previous study revealed that somatostatin analogs significantly lengthen the time to tumor progression in patients with metastatic midgut NETs (12). Therefore, immunohistochemical analysis of SSTR2a expression in NETs is required to examine the suitability for somatostatin receptor analog treatment. Although the metastatic rate is low in NET G1, the analysis of SSTR2a expression is useful for identifying the utility of an optional treatment for the unexpected metastasis of NETs.

Expression of the intermediate filaments, peripherin and $\alpha$-internexin, in NETs of the appendix and rectum has been previously reported $(13,14)$. We have previously characterized the expression patterns of neuronal intermediate filament 
A

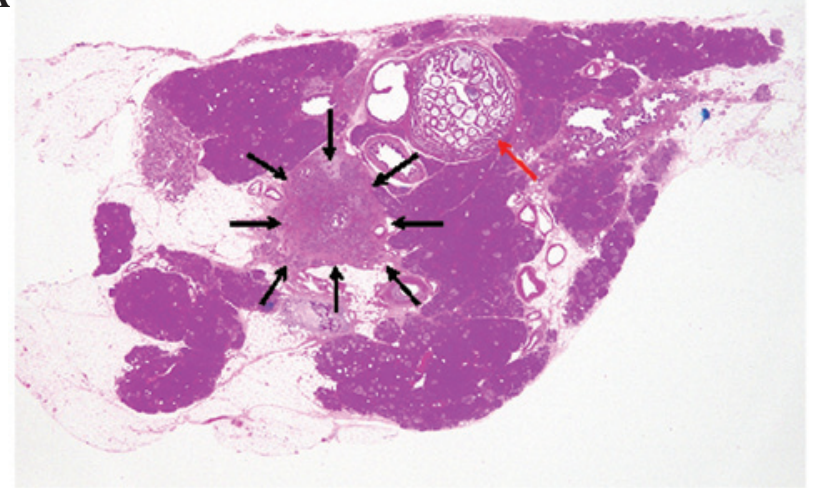

B

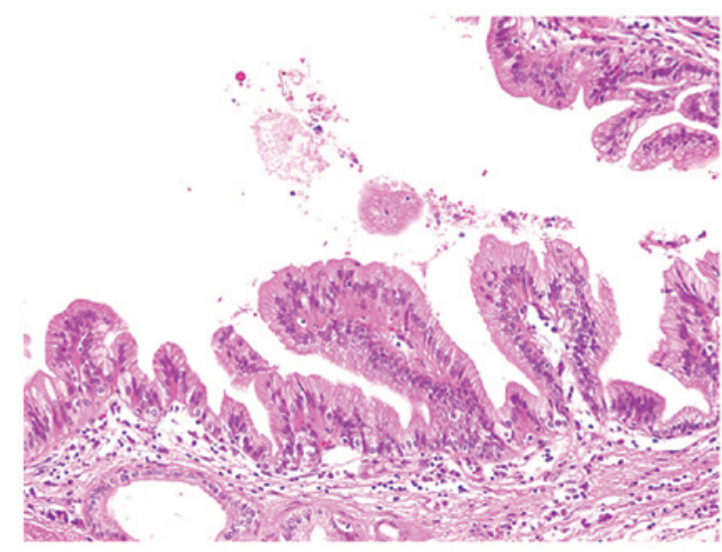

C

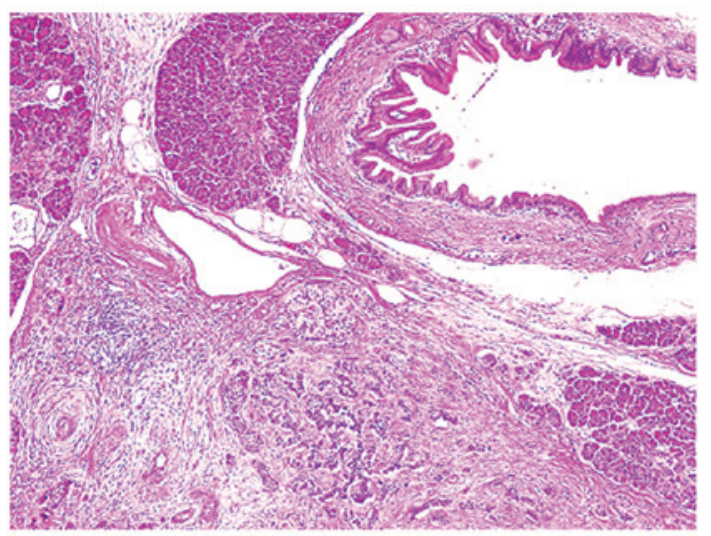

D

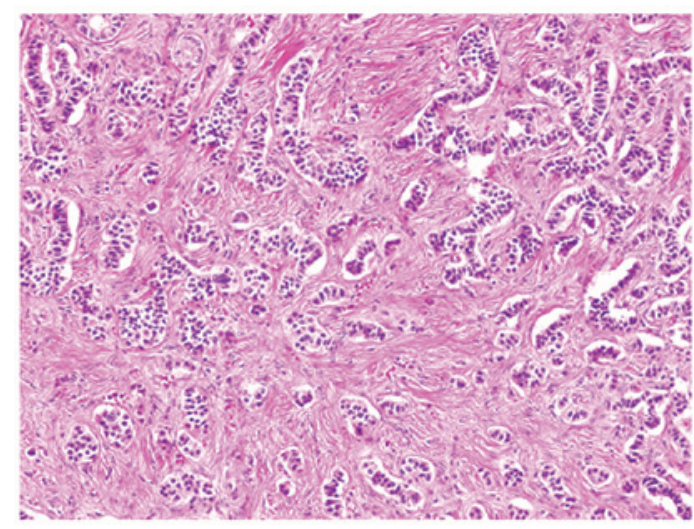

Figure 2. Histopathological findings. (A) Panoramic view of resected pancreas tissue showing dilated main pancreatic duct composed of papillary proliferation (intraductal papillary mucinous neoplasia: red arrow) and solid growth of the neuroendocrine tumor (black arrows) adjacent to main pancreatic duct (hematoxylin and eosin stain). (B) Main pancreatic duct with papillary proliferation of mucinous cells without atypia (hematoxylin and eosin stain, x200). (C and D) Neuroendocrine tumor showing trabecular growth of bland epithelial cells with fibrosis (hematoxylin and eosin stain, x40 and x200, respectively).
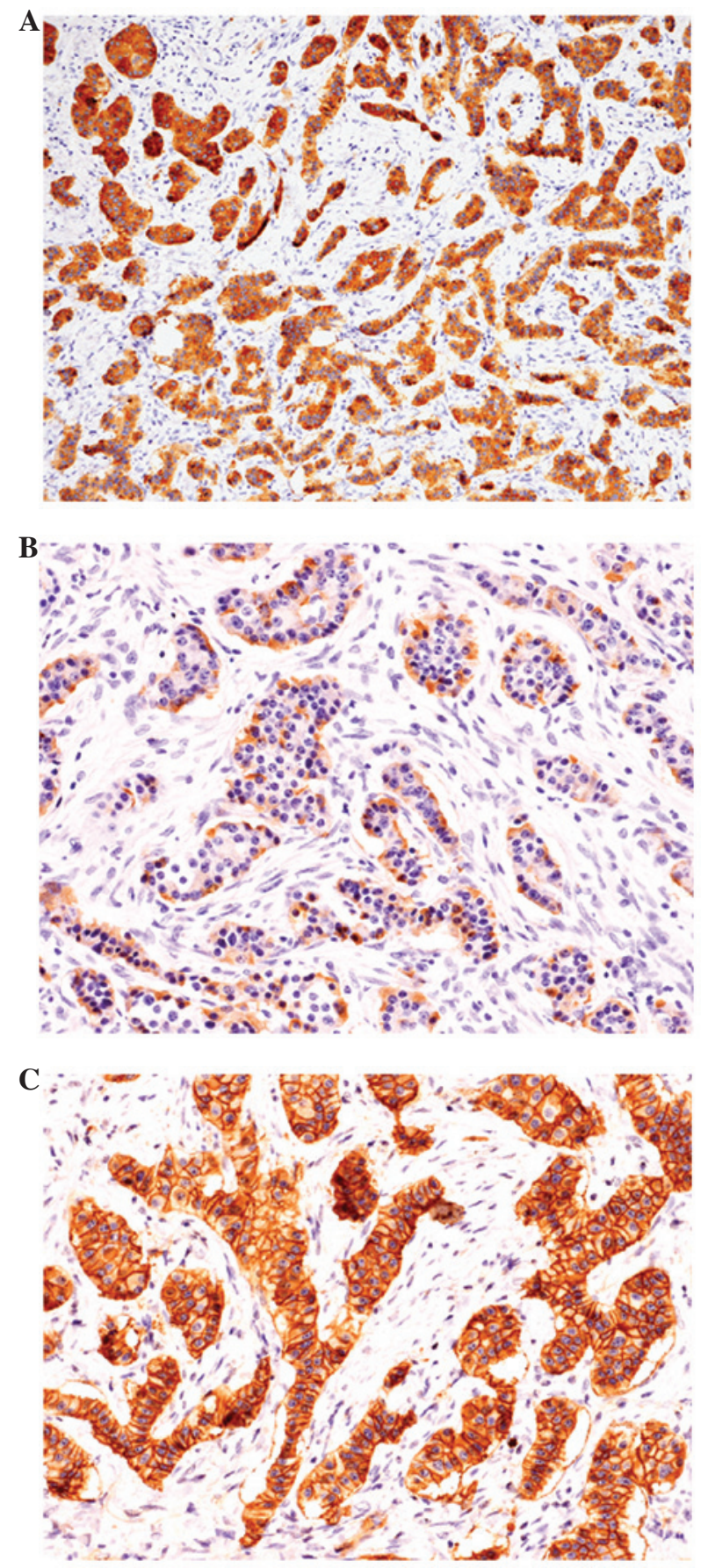

Figure 3. Immunohistochemical findings of the neuroendocrine tumor. (A) Synaptophysin is diffusely positive (x100). (B) Approximately half of the tumor cells were positive for glucagon (x200). (C) Tumor cells diffusely expressing somatostatin receptor type $2 \mathrm{a}$ in the cell membrane (x200).

proteins in the NETs of various organs $(13,14)$. While peripherin (a type III intermediate filament protein expressed in normal peripheral nerves) is expressed in all NET G1 of the rectum, the frequency of its expression is low in NET G2 of the rectum (13). By contrast, the expression of $\alpha$-internexin (a type IV intermediate filament protein normally found in the central nervous system) is observed in all NET G1 of the appendix and approximately half of rectal NET G1. All appendiceal NET G1 co-express peripherin and $\alpha$-internexin (14). Since neither peripherin nor $\alpha$-internexin expression was observed in this case of NET G1 of the pancreas, it appears 


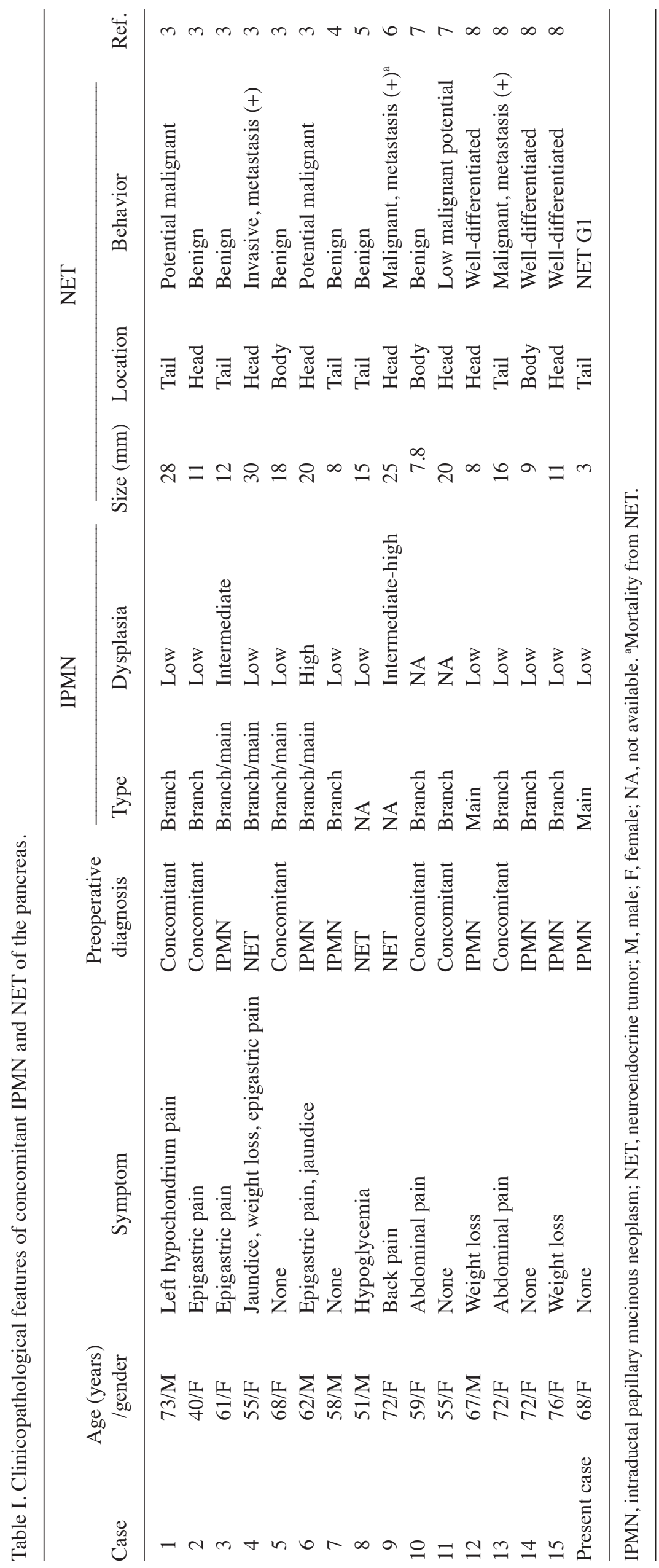


that intermediate filament protein expression varies with NET origin.

It is well known that IPMNs are associated with a high incidence of extrapancreatic malignancies, which proceed, coexist with or succeed IPMN (approximately 25-30\% of IPMN cases) (15-17). Colorectal and gastric carcinomas are the most common extrapancreatic carcinomas (15-17).

In conclusion, although patients with IPMN have a favorable prognosis with a 5-year survival rate of almost $100 \%$ (16), concomitant pancreatic NET and extrapancreatic malignancies may occur, therefore, systemic surveillance of extrapancreatic neoplasms and detection of concomitant NETs of the pancreas are necessary for patients with IPMN.

\section{References}

1. Adsay NV, Fukushima N, Furukawa T, et al: Intraductal neoplasms of the pancreas. In: World Health Organization Classification of Tumours of the Digestive System. Bosman FT, Carneiro F, Hruban RH and Theise ND (eds). IARC, Lyon, pp304-313, 2010.

2. Klimstra DS, Arnold R, Capella C, et al: Neuroendocrine neoplasms of the pancreas. In: World Health Organization Classification of Tumours of the Digestive System. Bosman FT, Carneiro F, Hruban RH and Theise ND (eds). IARC, Lyon, pp322-326, 2010

3. Marrache F, Cazals-Hatem D, Kianmanesh R, et al: Endocrine tumor and intraductal papillary neoplasm of the pancreas: a fortuitous association? Pancreas 31: 79-83, 2005.

4. Volante M, Brizzi MP, Faggiano A, et al: Somatostatin receptor type $2 \mathrm{~A}$ immunohistochemistry in neuroendocrine tumors: a proposal of scoring system correlated with somatostatin receptor scintigraphy. Mod Pathol 20: 1172-1182, 2007.

5. Goh BK, Ooi LL, Kumarasinghe MP, et al: Clinicopathological features of patients with concomitant intraductal papillary mucinous neoplasm of the pancreas and pancreatic endocrine neoplasm. Pancreatology 6: 520-526, 2006.

6. Zhao X, Stabile BE, Mo J, Wang J and French SW: Nesidioblastosis coexisting with islet cell tumor and intraductal papillary mucinous hyperplasia. Arch Pathol Lab Med 125; 1344-1347, 2001.
7. Stukavec J, Jirasek T, Mandys V, et al: Poorly differentiated endocrine carcinoma and intraductal papillary-mucinous neoplasm of the pancreas: Description of an unusual case. Pathol Res Pract 203: 879-884, 2007.

8. Larghi A, Stobinski M, Galasso D, Lecca PG and Costamagna G: Concomitant intraductal papillary mucinous neoplasm and pancreatic endocrine tumor: Report of two cases and review of the literature. Dig Liver Dis 41: 759-761, 2009.

9. Gill KR, Scimeca D, Stauffer J, et al: Pancreatic neuroendocrine tumors among patients with intraductal papillary mucinous neoplasms: real association or just a coincidence? JOP 10: 515-517, 2009.

10. Lamberts SW, Krenning EP and Reubi JC: The role of somatostatin and its analogs in the diagnosis and treatment of tumors. Endocr Rev 12: 450-482, 1991.

11. Reisine T and Bell GI: Molecular biology of somatostatin receptors. Endocr Rev 16: 427-442, 1995.

12. Rinke A, Müller H, Schade-Brittinger C, et al: Placebo-controlled, double-blind, prospective, randomized study on the effect of octreotide LAR in the control of tumor growth in patients with metastatic neuroendocrine midgut tumors: A report from the PROMID Study Group. J Clin Oncol 27: 4656-4663, 2009.

13. Ishida $M$, Kushima $R$, Chano $T$ and Okabe $H$ : Immunohistochemical demonstration of the type III intermediate filament peripherin in human rectal mucosae and well-differentiated endocrine neoplasms. Oncol Rep 18: 633-637, 2007.

14. Ishida M, Kushima R, Brevet M, Chatelain D and Okabe H: Co-expression of neuronal intermediate filaments, peripherin and $\alpha$-internexin in human well-differentiated endocrine neoplasms (carcinoid tumors) of the appendix. Mol Med Rep 1: 191-195, 2008.

15. Sugiyama M and Atomi Y: Extrapancreatic neoplasms occur with unusual frequency in patients with intraductal papillary mucinous tumors of the pancreas. Am J Gastroenterol 94: 470-473, 1999.

16. Sugiyama M,Suzuki Y, Abe N, Mori T and Atomi Y: Management of intraductal papillary mucinous neoplasm of the pancreas. J Gastroenterol 43: 181-185, 2008.

17. Ishida M, Egawa S, Kawaguchi K, et al: Synchronous and metachronous extrapancreatic malignant neoplasms in patients with intraductal papillary-mucinous neoplasm of the pancreas. Pancreatology 8: 577-582, 2008. 